\title{
Estimation of Influenza Incidence by Age in the 2011/12 Seasons in Japan using SASSy
}

\author{
Yasushi Ohkusa*1, Yoshinori Yasui ${ }^{1}$, Tamie Sugawara ${ }^{1}$, Nobuhiko Okabe ${ }^{2,1}$, Kiyosu \\ Taniguchi ${ }^{1}$ and Kazunori Oishi ${ }^{1}$
}

${ }^{1}$ IDSC,NIID, Shinjuku, Japan; ${ }^{2 K a w a s a k i ~ C i t y ~ I n s t i t u t e ~ f o r ~ P u b l i c ~ H e a l t h, ~ K a w a s a k i, ~ J a p a n ~}$

\section{Objective}

So far, it is difficult to show the incidence rate of influenza in the official sentinel surveillance in Japan. Hence we construct the system which record infectious diseases at schools, kindergartens, and nursery schools, and then can show the accurate incidence rate of influenza in children by age/grade.

\section{Introduction}

So as to develop more effective countermeasures against influenza, timely and precise information about influenza activity at schools, kindergartens, and nursery schools may be helpful. At the Infectious Diseases Surveillance Center of the National Institute of Infectious Diseases, a School Absenteeism Surveillance System (SASSy) has been in operation since 2009. SASSy monitors the activity of varicella, mumps, mycoplasma pneumonia, pharyngoconjunctival fever, hand-foot-mouth disease, influenza, and many other infectious diseases in schools. In 2010, SASSy was extended to the Nursery School Absenteeism Surveillance System (NSASSy). These systems record the number of absentees due to infectious diseases in each class of all grades of schools every day. As a powerful countermeasure to the pandemic flu of 2009, SASSy was activated in 9 prefectures, in which included more than 6000 schools, and it is gradually being adopted in other prefectures. As of February 2012, 18 prefectures and 4 big cities, which together comprised 15,700 schools (about $35 \%$ of all schools in Japan), utilized SASSy. NSASSy is used in more than 4100 nursery schools, which is about $18 \%$ of all nursery schools in Japan. Some studies of similar systems were performed in the UK (1), Hong Kong $(2)$, and the USA $(3,4)$, examined surveillance systems for monitoring infectious disease incidence, but the systems to construct in those studies do not operate nationwide like SASSy or NSASSy, and they cannot provide influenza incidence rates in children.

\section{Methods}

All schools, kindergartens, and nursery schools in the community, enter data of the absentees due to infectious diseases into the system every day, thereby providing real-time data regarding infectious diseases prevalent in schools, to the schools around, school boards, public health centers, local governments, and medical professionals. It analyzed data for the 2011/2012 season (from September 1, 2011 to March 31, 2012) mainly, but also two seasons (2010/2011 and 2011/2012) were compared in some prefectures. In total, 12 prefectures, which comprised $2,352,839$ children, were participated in $2011 / 2012$ season. In the 2010/2011 season, $1,795,766$ children of 9 prefectures were analyzed.

\section{Results}

The incidence rate in the first grade of elementary schools is the highest both in the two seasons. The highest incidence rate in this grade distributes from $17.8 \%$ to $40.3 \%$ in $2011 / 2012$ season, and from $11.0 \%$ to $30.7 \%$ in $2010 / 2011$ season.

\section{Conclusions}

This study proved SASSy and NSASSy are quite useful for monitoring of influenza outbreak in schools and it will be gold standard of surveillance for school children in Japan. The present study also showed incidence rate of influenza in children at schools, kindergartens, and nursery schools, and proved the highest incidence was in the first grade of the elementary school. This is the first finding using such the huge number of subjects, which is more than 2 million. The intervention targeting to the weak age/grade is necessary for effective countermeasure and control of influenza and other infectious diseases.

\section{Keywords}

Surveillance; Influenza; School Absenteeism

\section{Acknowledgments}

This paper is financial supported by Ministry Health, Labour and Welfare, Japan.

\section{References}

1) Schmidt WP, Pebody R, Mangtani P: School absence data for influenza surveillance: a pilot study in the United Kingdom, Eurosurveillance, Volume 15, Issue 3, 21 January 2010

2) Calvin K.Y. Cheng, Benjamin J. Cowling, Eric H.Y. Lau, Lai Ming Ho, Gabriel M. Leung, and Dennis K.M. Ip, Electronic School Absenteeism Monitoring and Influenza Surveillance, Hong Kong, Emerg Infect Dis. 2012 May

3) Buehler JW, Berkelman RL, Hartley DM, Peters CJ: Syndromic surveillance and bioterrorism-related epidemics. Emerg Infect Dis. 2003; 9:1197-204

4) Besculides M, Heffernan R, Mostashari F, Weiss D.:Evaluation of school absenteeism data for early outbreak detection, New York City. BMC Public Health. 2005 Oct 7;5:105

*Yasushi Ohkusa

E-mail: ohkusa@nih.go.jp 